\title{
TRABAJADORES MIGRANTES Y SEGURIDAD SOCIAL: APROXIMACIÓN NACIONAL E INTERNACIONAL A LOS MECANISMOS DE PROTECCIÓN QUE OTORGAN CONTINUIDAD A LAS PRESTACIONES
}

\author{
I PARTE
}

\author{
PABlo ARELLANo ORTIZ* \\ Universidad Austral
}

\begin{abstract}
RESUMEN: Este trabajo intenta desarrollar el problema de las migraciones y su relación con la protección de la seguridad social al trabajador migrante. De esta manera se intenta dar una visión global a este problema. En primer lugar, se desarrollarán algunos aspectos conceptuales que se consideran necesarios para la compresión del trabajo tales como: trabajador migrante y coordinación. En un segundo lugar, se estudia cuales son las normas internacionales relativas a la problemática en cuestión. Así se hace referencia a las normas sobre derechos humanos relativas al tema y además se hace un particular análisis de las normas de la OIT y los principios que emanan de estas. En un tercer lugar, se analiza la normativa nacional. Se estudia y categoriza a los distintos acuerdos bilaterales vigentes sobre la materia para luego hacer una referencia al Convenio Multilateral Iberoamericano de Seguridad Social. En este acápite se realiza una evaluación de la legislación nacional sobre migraciones y seguridad social. El trabajo termina con una serie de conclusiones en relación a la normativa tanto nacional como internaciones sobre la protección de seguridad social que se debe entregar al trabajador migrante.
\end{abstract}

Palabras clave: Seguridad social, trabajador migrante, coordinación, acuerdos bilaterales.

ABSTRACT: This paper attempts to develop the problem of migration and its relationship with the social security protection of the migrant worker. Thus, the author attempts to give an overview of this problem. First, it will develop some conceptual aspects that are considered necessary for the understanding of the work, such as migrant workers and coordination. In the second part, we study wich are the international standards on the issue in question. In this way, a reference to human rights standards relating to the subject is made, and also, makes a particular analysis of the ILO standards and principles arising from these. In a third, we analyze the national legislation. It examines and categorizes the various bilateral agreements on the matter and then makes a reference to the Latin American Multilateral Agreement on Social Security. In this section, an evaluation of the national migration laws and social security is made. The paper ends with some conclusions in relation to national and international regulations on social security protection that must be gevien to the migrant worker.

Key words: Social security, migrant worker, coordination, bilateral agreements.

\footnotetext{
Profesor de Derecho del Trabajo y de Seguridad Social, Universidad Austral de Chile. Doctor en Derecho, Université Paris Ouest Nanterre La Defense, France; Master 2 Recherche Droit Social et Droit de la Santé, Université de Paris X Nanterre, France; Master 2 Recherche Droit Social de la Université de Paris II Panthéon Assas, France. Abogado, Universidad de Concepción, Chile. Dirección postal: Universidad Austral de Chile, Facultad de Ciencias Jurídicas y Sociales, Campus Isla Teja S/N, Valdivia, Chile. email: pablo.arellano@uach.cl

Este trabajo forma parte de los proyectos Fondecyt Iniciación N 11121677, "La coordinación de sistemas de seguridad social: Estudio comparado Unión Europea e Iberoamérica” y del Proyecto DID No S-2012-44 Universidad Austral de Chile, "Los mecanismos de coordinación de sistemas de seguridad social: Europa e Iberoamérica”.
} 


\section{INTRODUCCIÓN}

La globalización es un fenómeno que -aunque no lo queramos- cada vez nos toca de más cerca. La doctrina del derecho social ya lleva bastante tiempo discutiendo sobre los efectos de esta en la protección de los derechos de los trabajadores ${ }^{1}$. Dentro de este contexto, uno de los aspectos que preocupa es la movilidad de los trabajadores en un contexto glo$\mathrm{bal}^{2}$. Que los trabajadores se desplacen de sus países e incluso cambien de continentes puede parecer una realidad lejana a nuestro país, pero, sin embargo, no se trata de un fenómeno del cual debemos aislarnos.

Además, como se ha señalado, "la movilidad internacional de mano de obra ha aumentado bajo las condiciones contemporáneas de globalización, al tiempo que se han acelerado los niveles de explotación y de desregulación"3. Así se ha aumentado los niveles de desprotección de los trabajadores, y ello ligado al movimiento migratorio.

Uno de los cambios de mayor alcance que han producido la globalización, el proceso de creciente interacción social y de interdependencia en las relaciones económicas es el cambio cuantitativo y cualitativo de los movimiento migratorios, de esta manera, se ha incluso hablado de una nueva era de las migraciones ${ }^{4}$. Nosotros creemos que no se trata de una nueva era de migraciones. Estas siempre han existido y es natural que las cifras aumenten. Lo que sucede es algo muy diferente: las migraciones se han convertido en un proceso cada vez más complejo. De migración para asentarse permanentemente se ha pasado a migraciones temporales ${ }^{5}$. Además, los migrantes han variado y se han convertido en un grupo muy variado: trabajadores temporales, subcontratados, trabajadores calificados, estudiantes, buscadores de asilo, refugiados políticos, etc.

De esta manera, los problemas que ha traído la migración hoy en día han llevado a sostener que "se requiere una comprensión mucho mayor del fenómeno actual de la migración, con objeto de que la comunidad internacional pueda idear los medios e instrumentos

\footnotetext{
Al respecto por ejemplo ver: LyON-CAEN, A.; URBAN, Q. (dir). Le droit du travail á l'épreuve de la globalisation. Dalloz "Thémes et commentaires", Paris, 2008. VIJ, R. (ed). Globalisation and Werlfare, a critical reader. Palgrave Macmillan, 2007. Moreau, Marie-Ange. Normes sociales, droit du travail et mondialisation- Confrontations et mutations, A droit ouvert. DallozSirey, Paris, 2006. LyON-CAEN, Gerard y Antoine. Droit social européen et international. $-8^{\mathrm{e}}$ édition-. Paris, France: Précis, Dalloz, 1993.

2 Sobre las cuestiones relativas a la mobilidad consultar, por ejemplo: MAUDET-BENDAHAN, Marilyn. La mobilité géographique du travailleur salarié au sein de l'Union européenne. Bibliothèque de droit social, Tome 57. Paris, L.G.D.J, 2012. MOREAU, Marie-Ange, op. cit. (n. 1). COURSIER, Philippe. Conflit de lois en matière de contrat de travail. Bibliothèque de droit privé, tome 230. París, Francia: L.G.D.J, 1993.

3 TARAN, Patrick; Geronimi, Eduardo. "Globalización y migraciones laborales: importancia de la protección”, en: Perspectivas sobre las migraciones laborales. 3 S, Programa de Migraciones Internacionales, Oficina Internacional del Trabajo, Ginebra, 2003, p. 1.

4 Rodríguez-PiÑero Bravo Ferrer, Miguel. "La OIT y los trabajadores migrantes”. En: Javillier, Jean-Claude; GerNIGON, Bernard (sous la direction de). Les normes internationales du travail: un patrimoine pour l'avenir. Mélanges en l'honneur de Nicolas Valticos. Geneva, Italie: Bureau International du Travail, 2004, p. 541.

5 Hirose, Kenichi; NiKAC, Milos y TAMAGNO, Edward. Social security for migrant workers. A right-based approach. International Labour Organization, Decent Work Technical Support Team, and Country Office for Central and Eastern Europe-Budapest, ILO, 2011, p. 1
} 
necesarios para una mejor gestión de la migración"6. No se pude seguir pensando en los migrantes como hace algunas décadas.

El número de migrantes alrededor del mundo ha sido estimado por experto de la OIT en 214 millones de persones, de los cuales 105 millones son trabajadores migrantes ${ }^{7}$, o sea, trabajadores que migran por razones de empleo. La OIT señala a su vez que lamentablemente las estadísticas sobre migraciones internacionales de muchos países son escasas y no tienen en cuenta (o solo en forma parcial) a las personas que se encuentran presentes en su territorio sin la debida documentación ${ }^{8}$. Este trabajo se concentrará en entregar protección y en particular protección de seguridad social a aquellos trabajadores migrantes que se desplazan en busca de empleo, aunque conscientes que su determinación cuantitativa en concreto es extremadamente difícil. Pero, lo primero que debemos preguntarnos es ¿cómo se les protegerá fuera de su país?

Como bien señala Rodríguez Piñeiro, como premisa previa a nuestra pregunta,

"la regulación de la entrada y permanencia de extranjeros en el territorio nacional para el acceso a empleos o actividades profesionales ha sido considerada tradicionalmente materia de soberanía estatal, correspondiendo al legislador nacional establecer requisitos, autorizaciones y controles para el acceso al territorio nacional, para su instalación en él, y, en su caso, para ejercer en el mismo una actividad laboral"9.

Sin embargo, "los extranjeros que se encuentran en un país extraño son titulares de los derechos que pertenecen a la persona en cuanto a tal, imprescindibles para la garantía de su dignidad, no sometidos a la condición de nacionalidad ni disponibles por el legislador" 10 . Así entonces las cosas, los migrantes poseen derechos en cuanto a su calidad de persona y como tal cada Estado debe entregarles una protección.

Por otro lado algunos han constatado la ausencia de un marco multilateral que proteja el movimiento transfronterizo de personas ${ }^{11}$. Ante esto se hace necesario recurrir a la normativa internacional que busca proteger a los trabajadores migrantes. Algunos de estos instrumentos dicen relación con derechos humanos y otros con normas propias del trabajo de la OIT. Pero tal como lo señala Rodríguez-Piñeiro pareciera necesario "nuevos instrumentos e intervenciones internacionales que permitan encauzar la emigración y asegurar una protección adecuada de los trabajadores migrantes" ${ }^{12}$. Reconociéndole en forma más

\footnotetext{
6 OIT. En busca de un compromiso equitativo para los trabajadores migrantes en la economía globalizada. Informe VI, Sexto punto del orden del día Conferencia Internacional del Trabajo, 92a reunión, 2004, p. 4. 
explícita al migrante un estatuto jurídico propio como persona con sus derechos humanos y sus derechos como trabajador ${ }^{13}$.

En lo que respecta a la protección que entrega la seguridad social al trabajador migrante, materia objeto de este trabajo, la situación es difícil de resolver. Desde el momento que al migrante se le somete a la ley interna, aplicándosele la regla de la territorialidad de la ley, se le aplica por ende la ley nacional en materia de seguridad social ${ }^{14}$. Pero ¿qué sucede con los derechos en curso de adquisición y con las cotizaciones aportadas en otros países? Para poder resolver estas cuestiones

"el derecho de seguridad social ha recurrido a los tratados internacionales. El sistema de tratados permite que los estados signatarios puedan, mediante la combinación de principios propios del derecho de los tratados con otros específicos de la seguridad social, desarrollar mecanismos de reconocimiento de los derechos en curso de adquisición de los trabajadores que deben prestar servicios en mas de un Estado durante su vida activa, y asegurar el pago de sus beneficios aunque cambie de domicilio" 15 .

Sin embargo, la complejización de la migración a que cada vez más trabajadores migrantes corren el riesgo de perder sus derechos a cobertura de seguridad social ${ }^{16}$.

Hoy en día, se puede constatar que el fenómeno migratorio como un fenómeno global que afecta también a la región latinoamericana y a nuestro país. Así, existe un movimiento importante de extranjero que llegan a trabajar a Chile así como un movimiento de los chilenos que se van a trabajar a otro países de América Latina o de otros continentes. Somos un país que ha firmado numerosos acuerdos bilaterales, por sobre otros países de la región, y además recientemente parte de un acuerdo regional sin precedentes en materia de protección de los derechos de seguridad social de los trabajadores migrantes.

Desde un punto de vista jurídico un análisis de esta problemática implica la necesidad de realizar un estudio de la normativa de seguridad social de los trabajadores migrantes visualizando para ello dos ámbitos: uno nacional y otro internacional. El primero, conlleva estudiar aspectos constitucionales atingentes a la inclusión de trabajadores migrantes en la normativa social, los convenios en materia de seguridad social suscritos por nuestro país y la legislación de seguridad social. El segundo, implica estudiar los instrumentos internacionales sobre derechos humanos de los migrantes y las normas específicas de la OIT sobre protección de derechos en materia de seguridad social que emanan de este organismo.

Comenzaremos este trabajo con el análisis de los instrumentos internacionales para luego analizar la normativa nacional, sin embargo creemos necesario revisar algunas cuestiones previas a fin de comprender de mejor manera el objeto de este estudio.

\footnotetext{
13 Ibid., p. 547.

14 Ibid., p. 556.

15 Cifuentes Lillo, Hugo. "Tratados internacionales de seguridad social: Convenios bilaterales”, en: Estudios en Homenaje al profesor William Thayer. Santiago, Chile: Sociedad Chilena de Derecho del Trabajo y Seguridad Social, 1998, p. 17.

16 Hirose, Kenichi; Nikac, Milos y Tamagno, Edward, op. cit. (n. 5), p. 2.
} 


\section{CUESTIONES PREVIAS}

La protección de seguridad social de un trabajador migrante es un tema bastante técnico y la cultura jurídica de cada país puede implicar soluciones distintas. De esta manera creemos necesario como paso previo al análisis de la normativa internacional como nacional, esclarecer tres ideas que consideramos claves para el entendimiento de la problemática de este trabajo. Desarrollaremos primero, el concepto de trabajador migrante; luego, qué se entiende por seguridad social, y, finalmente, explicaremos en qué consiste el mecanismo de coordinación de sistemas de seguridad social.

\subsection{QUÉ SE ENTIENDE POR TRABAJADOR MIGRANTE}

Determinar el objeto de la protección es una tarea ardua. Esto, debido a que al decir que nuestro objeto será el trabajador migrante puede producir una serie de problemas al precisar quiénes pueden estar incluidos en esta categoría. De hecho, muchas veces se suele asociar al trabajador migrante a otros tipos de situaciones las cuales pueden o no tratarse de un migrante, así, encontramos a trabajadores ilegales, trabajo forzado, trabajo infantil, etc. Es tanto el sentimiento de que la migración es algo malo que se la ha asociado con la delincuencia y con el terrorismo. Incluso se habla que la mano de obra migrante ocupa trabajos denominados sucios, peligrosos y difíciles (dirty, dangerous and dificult jobs) ${ }^{17}$. No es de extrañar entonces que estos trabajadores sean confinados al sector no estructurado, en el trabajo al negro o bajo condiciones de explotación ${ }^{18}$. Sectores en los cuales se encuentran excluidos de la protección que entregan los sistemas de seguridad social.

Debemos señalar también que al momento de caracterizar al migrante existe una cuestión de género. Los trabajos para migrantes son a menudo más apropiados para los hombres, lo que trae como resultado que las mujeres tengan un menor acceso a la migración legal ${ }^{19}$.

En cuanto a la definición de trabajador migrante esta la podemos encontrar en la Convención Internacional sobre la protección de los derechos de todos los trabajadores migratorios y de sus familiares, en su artículo 2 número 1 . Así se señala que "se entenderá por "trabajador migratorio" toda persona que vaya a realizar, realice o haya realizado una actividad remunerada en un Estado del que no sea nacional". Esta definición es bastante amplia ya que incluye a todos los trabajadores sin distinguir si se trata de aquellos que pertenecen a la economía informal o a la formal.

Además, esta norma nos entrega en su número 2 una serie de definiciones de diversas categorías de trabajadores migratorios de acuerdo con la naturaleza de sus prestaciones de servicios. Estas definiciones nos permiten determinar si se trata o no trabajadores migrantes propiamente tales. Así, esta norma nos señala:

\footnotetext{
TARAN, Patrick; Geronimi, Eduardo, op. cit. (n. 3), pp. 1 y 5.

8 TARAN, Patrick; Geronimi, Eduardo, op. cit. (n. 3), p. 8.

19 TARAN, Patrick; Geronimi, Eduardo, op. cit. (n. 3), p. 11.
} 
“2. a) Se entenderá por "trabajador fronterizo" todo trabajador migratorio que conserve su residencia habitual en un Estado vecino, al que normalmente regrese cada día o al menos una vez por semana;

b) Se entenderá por "trabajador de temporada" todo trabajador migratorio cuyo trabajo, por su propia naturaleza, dependa de condiciones estacionales y solo se realice durante parte del año;

c) Se entenderá por "marino", término que incluye a los pescadores, todo trabajador migratorio empleado a bordo de una embarcación registrada en un Estado del que no sea nacional;

d) Se entenderá por "trabajador en una estructura marina" todo trabajador migratorio empleado en una estructura marina que se encuentre bajo la jurisdicción de un Estado del que no sea nacional;

e) Se entenderá por "trabajador itinerante" todo trabajador migratorio que, aun teniendo su residencia habitual en un Estado, tenga que viajar a otro Estado u otros Estados por períodos breves, debido a su ocupación."

Las prestaciones de seguridad social se entregan generalmente en base a períodos de calificación, de actividad o de residencia. Además, la afiliación al sistema nacional de seguridad social puede ser un requisito para la obtención de beneficios. Así, entendiendo a estas prestaciones con estos obstáculos, debemos limitarnos entonces al ámbito de aplicación al migrante regular. O sea, aquel migrante en forma legal en busca de un empleo formal ${ }^{20}$. No obstante lo anterior, los regímenes no contributivos de seguridad pueden eventualmente entregar prestaciones a aquellos migrantes que no forman parte de la economía formal e incluso a aquellos migrantes ilegales o irregulares. Dejamos en claro que este trabajo se concentrará en los mecanismos que protegen al migrante regular, ya que es este tipo de migrante el que se encuentra, por lo general, en los instrumentos internacionales de coordinación de sistemas de seguridad social.

\subsection{QUÉ SE ENTIENDE POR SEGURIDAD SOCIAL}

Una segunda cuestión previa importante dice relación con determinar a que nos referimos con el término seguridad social ${ }^{21}$. Sobre todo debido a que en el contexto de las migraciones podemos encontrarnos ante variadas concepciones en función de la legislación local de cada país.

En general, podemos señalar que para justificar la existencia de un derecho a la seguridad social, se mencionaron varias razones ${ }^{22}$ : la seguridad en el empleo, la seguridad de los

20 Hirose, Kenichi; Nikac, Milos y TAmagno, Edward, op. cit. (n. 5), p. 2.

21 Sobre la discusión sobre el concepto de seguridad social ver: ARELLANO ORTIZ, Pablo. Universalismo e Individualismo en el derecho chileno de pensiones. Santiago, Chile: Editorial Librotecnia, 2012. CifuENTES LiLlo, Hugo. "Seguridad Social: Conceptos, definiciones y sistema de protección social en Chile", en: Homenaje al profesor Alfredo Bowen H. Anuario de derecho del Trabajo y Seguridad Social n ${ }^{\circ}$. Santiago, Chile: Sociedad Chilena de Derecho del Trabajo y de la Seguridad Social, 2000, pp. 9-22. Novoa Fuenzalida, Patricio. Derecho de la Seguridad Social. Santiago, Chile: Editorial Jurídica de Chile, 1977.

22 LaRoque, P. «De l'assurance à la Sécurité Sociale, l'expérience française», en: Rev. Inter. Trav. vol. LVII, n 6, France, París, 1948, p. 623 et. ss. 
ingresos, la seguridad de la capacidad de trabajo, la seguridad por medio de la asignación de ingresos de reemplazo. Todas ellas resultan de vital importancia tanto para el residente de un país como para el migrante, emigrante e inmigrante.

La OIT en el Informe sobre el Trabajo en el Mundo del año 2000, considera como seguridad social la protección que una sociedad asegura a sus miembros mediante una serie de medidas públicas; entre las cuales incluye ${ }^{23}$ :

"los seguros sociales, la ayuda social o asistencia social y los sistemas universales, a saber: la seguridad social en cuanto a protección que la sociedad entrega a sus miembros a través de una serie de medidas públicas para compensar la ausencia o la reducción substancial de ingresos del trabajo resultante de diversas eventualidades (por ejemplo la enfermedad, la maternidad, los accidentes de trabajo, la cesantía, la invalidez o el fallecimiento); para otorgar a las personas prestaciones de salud, y para otorgar prestaciones a las familias que tengan niños" 24 .

Por su parte el Departamento de Seguridad Social de la OIT definió recientemente el concepto de seguridad social como aquel que:

"abarca todas las medidas relacionadas con las prestaciones, en efectivo o en especie, encaminadas a garantizar una protección en determinados casos, como por ejemplo:

- Falta de ingresos laborales (o ingresos laborales insuficientes) debido a enfermedad, discapacidad, maternidad, accidentes de trabajo, desempleo, vejez o muerte de un miembro de la familia;

- $\quad$ Falta de acceso o acceso a precios excesivos a la asistencia médica;

- $\quad$ Apoyo familiar insuficiente, en particular para los hijos y adultos a cargo;

- Pobreza generalizada y exclusión social.

Los sistemas de seguridad social pueden ser de carácter contributivo (seguro social) o de carácter no contributivo" 25 .

Una definición consensual de la noción de seguridad social a nivel internacional puede ser establecida de la manera siguiente: un grupo de instituciones, medidas, derechos y

\footnotetext{
23 KUPTSCH, C. «La privatisation dans la sécurité sociale: un slogan aux multiples facettes- Remarques sur le concept de privatisation en fonction du contexte», In: SCHEIL-ADLUNG, X. Construire la sécurité sociale: la privatisation en question. Francia: AISS, Peter Lang SA, Bern, 2001, p. 15.

24 OIT. Rapport sur le travail dans le monde 2000: sécurité du revenu et protection sociale dans un monde en mutation. Commission de l'emploi et de la politique sociale. Conférence Internationale du Travail, 79 session, Genève, 2000.

25 OIT. Seguridad social para la justicia social y una globalización equitativa. Discusión recurrente sobre la protección social (seguridad social) en virtud de la Declaración de la OIT relativa a la justicia social para una globalización equitativa, Informe VI, Sexto punto del orden del día, Conferencia Internacional del Trabajo, 100.a reunión, 2011.
} 
obligaciones cuyo objeto principal es entregar, o tiende a otorgar, según reglas específicas, una garantía a los ingresos y a la salud de los individuos miembros de una sociedad ${ }^{26}$.

Sin lugar a dudas se puede notar una evolución en el concepto de seguridad social. Ya no se hace una referencia explícita a los mecanismos de seguros sociales y de asistencia social, ni tampoco a los riesgos a proteger. Lo más importante es que cada vez se habla menos de derecho a prestaciones. Para los efectos de este trabajo ocuparemos una definición que creemos que comprende en forma completa y con alcance global el sentido de la seguridad social. Así, nosotros hemos definido a:

"la seguridad social en cuanto rama del derecho, como el conjunto integrado de principios, de normas e instituciones de ordenación estatal, destinadas a otorgar protección a las personas frente a las contingencias o estados de necesidad (riesgos sociales) a través de prestaciones (transferencias) en dinero o en especie, de financiamiento contributivo o no contributivo, cuya administración puede ser pública y/o privada, debiendo el Estado asegurar un mínimo de protección a su población" 27 .

\subsection{EN QUÉ CONSISTE LA COORDINACIÓN DE SISTEMAS DE SEGURIDAD SOCIAL}

La coordinación tiene una estrecha relación con los procesos de integración política y económica entre dos o más países. Este proceso se puede lograr a través de diferentes tipos de acuerdos bilaterales o multilaterales. En ambos casos la doctrina ha manifestado su aceptación por una serie de principios básicos que se encuentran sentados en normas de la OIT. Rodríguez-Piñeiro señala en relación a las normas de la OIT que estas:

"en su conjunto, y en la medida en que los convenios de la OIT se ratifiquen por un número significativos de Estados, producen un cierto proceso de armonización de los sistemas laborales nacionales, pero sin implicar una “internacionalización” de la dimensión territorial nacional que se predica del derecho del trabajo" 28 .

\section{Como bien señala Gumucio:}

"en los procesos de integración, se plantean distintos grados de acercamiento en materia de legislación laboral y de seguridad social. Por una parte, el establecimiento de mecanismos intermediarios de coordinación para posibilitar la interconexión o funcionamiento vinculado entre disposiciones de los distintos ordenamientos nacionales de los países que lo integran"29.

\footnotetext{
26 KUlKe, U.; CiCHON, C.; PALK, K. "Changing tides: a revival of a right-based approach to social security". En: LANGENDONCK, J. van (Ed.). The Right to Social Security. Inglaterra, Londres: Intersentia, Antwerp- Oxford, 2007, p. 13.

27 Cifuentes Lillo, Hugo; Arellano Ortiz, Pablo. "Derecho a la Seguridad Social y la protección por pensiones de vejez, invalidez y sobrevivencia en Chile”, en: RojAs MiN̄o, Irene (Coordinadora). Cincuenta años de la Sociedad Chilena de Derecho del Trabajo y de la Seguridad Social: Homenaje. Santiago, Chile: Sociedad Chilena de Derecho del Trabajo y de la Seguridad Social, AbeledoPerrot LegalPublishing, 2011, pp. 186.

28 Rodríguez-Piñero Bravo Ferrer, Miguel, op. cit. (n. 4), p. 549.

29 Gumucio R, J. "Chile en el Mercosur: armonización de sistemas de Seguridad Social”, en: Revista Laboral Chilena, enero, Santiago, Chile, 1997, p. 59.
} 
Además de la coordinación "la integración puede suponer un esfuerzo de convergencia legislativa que puede dirigirse a la meta más ambiciosa de la unificación o a las más modestas de una armonización que elimine las diferencias que en mayor grado dificultan la integración" 30 .

Tomando en lo anterior en cuenta es que debemos señalar que existe una marcada diferencia entre coordinación y armonización. Si bien se trata de dos tipos de técnicas que permiten una cierta uniformización de legislaciones, en la armonización se intenta que los involucrados adopten la misma norma jurídica; en la coordinación, en cambio, esto no se produce y cada país conserva su propia autonomía de su legislación. Entendidos así estos conceptos, resulta difícil que en materia de seguridad social se acuerde una armonización ya que ello implicaría que los países perderían injerencia su propia cultura de protección de riesgos sociales.

Para los efectos de este trabajo seguiremos lo señalado en una reciente publicación de la OIT, la cual señala que la:

"coordinación significa establecer mecanismo a través de los cuales los sistemas de seguridad social de diferentes países pueden trabajar juntos para lograr en conjunto objetivos acordados -en particular para asegurar que los trabajadores migrantes y los miembros de sus familias tengan protección tan completa y continua como sea posible- mientras, al mismo tiempo, mantenga y respete las definiciones y reglas de cada sistema por separado. Coordinación no involucra reemplazar las diferentes definiciones y reglas de cada sistema con definiciones y reglas comunes, lo que es usualmente referido como armonización"31.

\section{LA REGLAMENTACIÓN DE SEGURIDAD SOCIAL DEL TRABAJADOR MIGRANTE A NIVEL INTERNACIONAL}

La regulación de la situación de los migrantes ha tomado diversas formas. Podemos encontrar que diferentes tipos de medidas se han adoptado a nivel internacional para proteger al trabajador migrante.

De entre todos los instrumentos internacional la Convención internacional sobre la protección de los derechos de todos los trabajadores migratorios y de sus familiares más el convenios 97 y el convenio 143 OIT son considerados como los instrumentos claves en esta materia. Se estima que:

"estos tres instrumentos en su conjunto proveen una definición y una base éticojurídica para la política y práctica nacionales respecto de los trabajadores migrantes extranjeros y los miembros de sus familias, así como herramientas para alentar a los Estados a adoptar y mejorar una legislación nacional conforme a las normas internacionales. No se trata solo de instrumentos de derechos humanos sino que numerosas

\footnotetext{
$30 \quad$ Ibid., p. 59.

31 Hirose, Kenichi; NiKaC, Milos y Tamagno, Edward, op. cit. (n. 5), p. 24.
} 
disposiciones sientan principios para la elaboración de una política nacional y para la consulta y cooperación interestatales con miras a la formulación de una política sobre migraciones laborales, al intercambio de información, al suministro de información a los migrantes, a la gestión de retornos ordenados o a la reintegración”32.

Además de estos instrumentos que son el marco general existen otros. Estos son los acuerdos bilaterales y multilaterales que adoptan los países siguiendo las directrices que se establecen en este marco general.

En este apartado haremos primero una somera revisión de cuáles son los instrumentos internacionales de derechos humanos. En seguida analizaremos los instrumentos de la OIT relativos a migraciones, y en particular los relativos a la seguridad social del migrante. Continuaremos con una descripción de los principios generales establecidos por instrumentos internacionales de protección de los derechos de seguridad social. Luego, explicaremos como la coordinación opera en instrumentos bilaterales y multilaterales. Finalmente, realizaremos algunas reflexiones sobre la situación de estos instrumentos internacionales.

\subsection{NORMAS DE DERECHOS HUMANOS}

La Convención Internacional sobre la Protección de los Derechos de todos los Trabajadores Migratorios y de sus Familiares de 1990 constituye el instrumento más importante en materia de derechos humanos. Al respecto existe una campaña mundial en busca de su ratificación y respecto de los derechos consagrados en ella ${ }^{33}$.

Este instrumento no es el único instrumento internacional sobre derechos humanos que contiene disposiciones relativas al trabajador migrante. Hacer un análisis detallado de cada instrumento resultaría un trabajo demasiado extenso. Sin embargo para los efectos de este trabajo interesa determinar cuáles son estos instrumentos y si nuestro país los ha ratificado. A continuación incluimos un cuadro que incluye dicha información.

\begin{tabular}{l|l|l}
\hline \multicolumn{1}{c|}{$\begin{array}{c}\text { Instrumento especifico de derecho } \\
\text { humanos sobre migraciones }\end{array}$} & \multicolumn{1}{|c}{ Adopción } & \multicolumn{1}{|c}{$\begin{array}{c}\text { Firma / Ratificación por } \\
\text { Chile }\end{array}$} \\
\hline $\begin{array}{l}\text { Convención internacional sobre la } \\
\text { protección de los derechos de todos } \\
\text { los trabajadores migratorios y de sus } \\
\text { familiares }\end{array}$ & $\begin{array}{l}\text { Adoptada por la Asamblea General } \\
\text { en su resolución 45/158, de 18 de } \\
\text { diciembre de 1990 (entro en vigencia } \\
\text { el 1 de julio de 2003) }\end{array}$ & $\begin{array}{l}24 \text { septiembre 1993 / 21 } \\
\text { marzo de 2005 }\end{array}$ \\
\hline $\begin{array}{l}\text { Otros instrumentos sobre derecho bu- } \\
\text { manos atingentes a migraciones }\end{array}$ & Adopción & $\begin{array}{l}\text { Firma / Ratificación por } \\
\text { Chile }\end{array}$ \\
\hline $\begin{array}{l}\text { Convención Internacional sobre la la } \\
\text { Eliminación de todas las Formas de } \\
\text { Discriminación Racial }\end{array}$ & $\begin{array}{l}\text { Adoptada y abierta a la firma y rati- } \\
\text { ficación por la Asamblea General en } \\
\text { su resolución 2106 A (XX), de 21 } \\
\text { de diciembre de 1965. Entrada en } \\
\text { vigor: 4 de enero de 1969, de con- } \\
\text { formidad con el artículo 19 }\end{array}$ & $\begin{array}{l}3 \text { octubre 1966 / 20 oc- } \\
\text { tubre 1971 }\end{array}$ \\
\hline
\end{tabular}

32 Taran, Patrick; Geronimi, Eduardo, op. cit. (n. 3), p. 16.

33 Sobre esta campaña ver http://migrantsrights.org 


\begin{tabular}{|c|c|c|}
\hline $\begin{array}{l}\text { Instrumento especifico de derecho } \\
\text { humanos sobre migraciones }\end{array}$ & Adopción & $\begin{array}{l}\text { Firma / Ratificación por } \\
\text { Chile }\end{array}$ \\
\hline $\begin{array}{l}\text { Convención sobre la eliminación de } \\
\text { todas las formas de discriminación } \\
\text { contra la mujer }\end{array}$ & $\begin{array}{l}\text { Adoptada y abierta a la firma y ratifi- } \\
\text { cación, o adhesión, por la Asamblea } \\
\text { General en su resolución } 34 / 180 \text {, de } \\
18 \text { de diciembre de } 1979 \text {. Entrada en } \\
\text { vigor: } 3 \text { de septiembre de } 1981 \text {, de } \\
\text { conformidad con el artículo } 27 \text { (1) }\end{array}$ & $\begin{array}{l}17 \text { julio } 1980 / 7 \text { diciembre } \\
1989\end{array}$ \\
\hline $\begin{array}{l}\text { Pacto Internacional de Derechos } \\
\text { Económicos, Sociales y Culturales }\end{array}$ & $\begin{array}{l}\text { Adoptado y abierto a la firma, rati- } \\
\text { ficación y adhesión por la Asamblea } \\
\text { General en su resolución } 2200 \text { A } \\
\text { (XXI), de } 16 \text { de diciembre de } 1966 \text {. } \\
\text { Entrada en vigor: } 3 \text { de enero de } 1976 \text {, } \\
\text { de conformidad con el artículo } 27 \text {. }\end{array}$ & $\begin{array}{l}16 \text { septiembre } 1969 / 10 \\
\text { febrero } 1972\end{array}$ \\
\hline $\begin{array}{l}\text { Pacto Internacional de Derechos Ci- } \\
\text { viles y Políticos }\end{array}$ & $\begin{array}{l}\text { Adoptado y abierto a la firma, rati- } \\
\text { ficación y adhesión por la Asamblea } \\
\text { General en su resolución } 2200 \text { A } \\
\text { (XXI), de } 16 \text { de diciembre de } 1966 \text {. } \\
\text { Entrada en vigor: } 23 \text { de marzo de } \\
1976 \text {, de conformidad con el ar- } \\
\text { tículo } 49 \text { Lista de los Estados que } \\
\text { han ratificado el pacto } \\
\text { Declaraciones y reservas }\end{array}$ & $\begin{array}{l}16 \text { septiembre } 1969 / 10 \\
\text { febrero } 1972\end{array}$ \\
\hline $\begin{array}{l}\text { Convención sobre los Derechos del } \\
\text { Niño }\end{array}$ & $\begin{array}{l}\text { Adoptada y abierta a la firma y rati- } \\
\text { ficación por la Asamblea General en } \\
\text { su resolución } 44 / 25 \text {, de } 20 \text { de no- } \\
\text { viembre de } 1989 \text {. Entrada en vigor: } \\
2 \text { de septiembre de } 1990 \text {, de confor- } \\
\text { midad con el artículo } 49\end{array}$ & $\begin{array}{l}26 \text { enero } 1990 / 13 \text { agosto } \\
1990\end{array}$ \\
\hline $\begin{array}{l}\text { Protocolo para prevenir, reprimir y } \\
\text { sancionar la trata de personas, espe- } \\
\text { cialmente mujeres y niños, que com- } \\
\text { plementa la Convención de las } \mathrm{Na} \text { - } \\
\text { ciones Unidas contra la Delincuencia } \\
\text { Organizada Transnacional }\end{array}$ & $\begin{array}{l}\text { Adoptada y abierta a la firma y rati- } \\
\text { ficación por la Asamblea General en } \\
\text { su resolución } 55 / 25 \text {, de } 15 \text { de no- } \\
\text { viembre de } 2000 \text {. Entrada en vigor: } \\
25 \text { de diciembre de } 2003 \text {. }\end{array}$ & $\begin{array}{l}8 \text { agosto } 2002 \text { / } 29 \text { noviem- } \\
\text { bre } 2004\end{array}$ \\
\hline $\begin{array}{l}\text { Protocolo contra el tráfico ilícito de } \\
\text { migrantes por tierra, mar y aire, que } \\
\text { complementa la Convención de las } \\
\text { Naciones Unidas contra la Delin- } \\
\text { cuencia Organizada Transnacional }\end{array}$ & $\begin{array}{l}\text { Adoptada por resolución A/ } \\
\text { RES/55/25 de } 15 \text { de noviembre de } \\
2000 \text { en la } 55 \text { Asamblea General. } \\
\text { Entrada en vigor: } 28 \text { de enero de } \\
2004 \text {. }\end{array}$ & $\begin{array}{l}8 \text { agosto } 2002 \text { / } 29 \text { noviem- } \\
\text { bre } 2004\end{array}$ \\
\hline
\end{tabular}

\subsection{NORMAS INTERNACIONALES DE LA OIT}

La OIT desde muy temprano ha adoptado una serie de instrumentos que protegen al trabajador migrante ${ }^{34}$. Los instrumentos que se refieren a los trabajadores migrantes son

34 TARAn, Patrick; Geronimi, Eduardo, op. cit. (n. 3), pp. 6 y ss. 
los dos Convenios específicos (números 97 y 143) así como las recomendaciones que los acompañan, y los instrumentos de la OIT sobre los principios y derechos fundamentales ${ }^{35}$, así como en principio todas las demás normas de la OIT. La OIT ha adoptado normas que versan específicamente sobre los trabajadores migrantes en dos contextos políticos muy diferentes ${ }^{36}$ : el primero en 1949, después de la Segunda Guerra Mundial, y más tarde, en 1975, a raíz de la crisis del petróleo de 1973.

Los instrumentos del primer período se debieron, entre otras razones, al interés por facilitar el movimiento del excedente de mano de obra de Europa a otras partes del mundo. Las disposiciones del Convenio sobre los trabajadores migrantes (revisado) $\mathrm{N}^{\circ}$ 97, 1949 y de la Recomendación sobre los trabajadores migrantes (revisada) № 86, 1949 complementaria se centran en las normas aplicables a la contratación de trabajadores migrantes para el empleo y a sus condiciones de trabajo. Taran y Geronimi nos señalan que este convenio:

"sienta las bases para la igualdad de tratamiento entre trabajadores nacionales y extranjeros en situación regular en cuestiones como los procedimientos de contratación, las condiciones de vida y de trabajo, el acceso a la justicia, y la reglamentación sobre impuestos y seguridad social. Establece disposiciones sobre las condiciones de contratación, la participación de migrantes en la formación y promoción profesionales, la reunificación familiar, el despido injustificado o la expulsión, así como otras medidas destinadas a regular el proceso migratorio en su conjunto" 37.

Por su parte, en el período de 1975 a los gobiernos les preocupaba cada vez más el desempleo y el aumento de la migración irregular, por lo que dejó de hacerse hincapié en facilitar la migración del excedente de mano de obra y la atención pasó a centrarse en controlar los flujos migratorios. A juicio de Taran y Geronimi en este momento "la comunidad internacional empezó a tomar en cuenta el aumento de la migración irregular. Sus dos principales objetivos son: 1) regular los flujos migratorios, eliminar la migración clandestina y luchar contra las actividades de tráfico y de trata de personas; y 2) facilitar la integración de los migrantes en las sociedades de acogida" 38 . De esta manera, la OIT adopta en este período dos nuevas normas: el Convenio sobre los trabajadores migrantes (disposiciones complementarias) $\mathrm{N}^{\circ} 143,1975$ y la Recomendación sobre los trabajadores migrantes $\mathrm{N}^{\circ} 151$, 1975. Estos instrumentos constituyen el primer intento multilateral de abordar el problema de los migrantes irregulares y también de propugnar sanciones contra los «traficantes» de personas.

En el Convenio $N^{\circ} 143$, la OIT vuelve a señalar que los Estados Miembros tienen la obligación general de respetar los derechos humanos fundamentales de todos los trabajado-

\footnotetext{
35 Sobre los principios y derechos fundamentales ver lo que hemos escrito en: ARELLANO ORTIZ, Pablo. "La conformidad de la legislación chilena a las normas internacionales del trabajo de la OIT", en: Revista de Derecho de la Universidad Católica de la Santísima Concepción, Concepción, Chile, 2011, pp. 39-60.

36 OIT, En busca de un compromiso equitativo para los trabajadores migrantes en la economía globalizada, Informe VI, Sexto punto del orden del día Conferencia Internacional del Trabajo, 92. ${ }^{a}$ reunión, 2004, p.86.

37 TARAN, Patrick; Geronimi, Eduardo, op. cit. (n. 3), p. 13.

38 Ibid.
} 
res migrantes. Además se establece que no solo deberán gozar de igualdad de trato (como se prevé en el Convenio $\mathrm{N}^{\circ}$ 97) sino también de la igualdad de oportunidades, por ejemplo en lo tocante al acceso al empleo, los derechos sindicales, los derechos culturales y las libertades individuales y colectivas.

En relación a la protección que entrega la seguridad social a los migrantes, como ya lo hemos señalado, la situación es compleja debido a que los derechos a las prestaciones son en función de la duración del empleo, de las cotizaciones o de la residencia, por lo que pueden perder su derecho a las prestaciones de la seguridad social en su país de origen debido a una ausencia prolongada y estar al mismo tiempo sometidos a condiciones restrictivas en el país de destino, en lo que atañe a su protección por el sistema nacional de seguridad social. La OIT señalado que para los trabajadores migrantes es particularmente importante ${ }^{39}$ : a) tener el mismo acceso a la cobertura y el mismo derecho a las prestaciones que los nacionales; b) conservar los derechos adquiridos al irse del país (incluida la exportación de las prestaciones), y c) poder acumular derechos adquiridos en países diferentes.

De esta manera, las diversas normas internacionales de la OIT buscan de alguna manera poder darle una respuesta al migrante en relación a los tres puntos enunciados.

En todas las normas de la OIT relativas a la seguridad social vigentes se define el grado personal de cobertura sin tener en cuenta la nacionalidad y en casi todas ellas hay cláusulas similares sobre la igualdad de trato entre los trabajadores nacionales y los extranjeros en el país de inmigración. No obstante, en el Convenio sobre la igualdad de trato (accidentes del trabajo) $\mathrm{N}^{\circ} 19,1925$, se establece específicamente el derecho a la igualdad de trato de los trabajadores extranjeros de cualquier otro Estado que haya ratificado el Convenio, en lo relativo a la indemnización en caso de accidentes de trabajo. Este Convenio autoriza además la exportación de las prestaciones de los trabajadores extranjeros amparados por el Convenio, pero solamente cuando el Estado ratificante consiente la exportación de las prestaciones en el caso de sus propios nacionales.

En el Convenio sobre la igualdad de trato (seguridad social) $\mathrm{N}^{\circ} 118,1962$, se estipula el derecho a la igualdad de trato en las nueve ramas clásicas de la seguridad social. En cada una de las nueve ramas que acepte el Estado parte se compromete a conceder en su territorio a los nacionales de cualquier otro Estado que haya ratificado el Convenio la igualdad de trato con sus propios nacionales. Además, estipula que todo Estado parte tiene que velar por el suministro de prestaciones en el extranjero en una rama concreta para sus propios nacionales y para los de cualquier otro Estado que haya aceptado las obligaciones del Convenio en la misma rama, cualquiera que sea el lugar de residencia de los beneficiarios.

Ambos convenios, el $\mathrm{n}^{\circ} 19$ y el $\mathrm{n}^{\circ} 118$, establecen disposiciones que permiten señalar que se consagrar el principio de la reciprocidad en relación a las prestaciones de seguridad social para los trabajadores migrantes.

En otros instrumentos de la OIT sobre la seguridad social también se incluyen cláusulas especiales contra la discriminación. El Convenio sobre la seguridad social (norma mínima) $n^{\circ} 102$, 1952, el cual sienta los principios generales para todo sistema de seguridad social, consagra su Parte XI totalmente a la igualdad de trato de los residentes no nacionales.

39 OIT. En busca de un compromiso... (n. 6), p. 88. 
En su artículo 68, que se aplica a todas las ramas de la seguridad social, se estipula que los nacionales y los no nacionales deben tener los mismos derechos a la seguridad social, pero con cierta flexibilidad, que permita la exclusión de no nacionales cuando las prestaciones o una parte de las mismas se abonen en su totalidad con cargo a fondos públicos. A saber dicha disposición señala:

“1. Los residentes no nacionales deberán tener los mismos derechos que los residentes nacionales. Sin embargo, podrán prescribirse disposiciones especiales para los no nacionales y para los nacionales nacidos fuera del territorio del Miembro, en lo que respecta a las prestaciones o partes de prestaciones financiadas exclusivamente o de manera preponderante con fondos públicos, y en lo que respecta a los regímenes transitorios.

2. En los sistemas de seguridad social contributivos cuya protección comprenda a los asalariados, las personas protegidas que sean nacionales de otro Miembro que haya aceptado las obligaciones de la parte correspondiente del Convenio deberán tener, respecto de dicha parte, los mismos derechos que los nacionales del Miembro interesado. Sin embargo, la aplicación de este párrafo podrá estar condicionada a la existencia de un acuerdo bilateral o multilateral que prevea la reciprocidad".

Por su parte, el Convenio sobre la conservación de los derechos en materia de seguridad social $\mathrm{N}^{\circ}$ 157, 1982 y la Recomendación sobre la conservación de los derechos en materia de seguridad social $N^{\circ} 163,1983$, constituyen una evolución en las normas de la OIT en esta materia, formando un sistema internacional para el mantenimiento de los derechos adquiridos, o en curso de adquisición, de los trabajadores que trasladen su residencia de un país a otro, y para asegurar una protección real de las prestaciones adquiridas en el extranjero cuando regresen a su país de origen. Este Convenio establece mecanismos para garantizar la conservación de los derechos adquiridos a los nacionales de otros Estados partes del Convenio en toda rama de la seguridad social para la cual esos Estados tengan una legislación vigente. Además, el Convenio menciona como mecanismo para la consecución de sus objetivos la firma de acuerdos bilaterales o multilaterales de seguridad social. La Recomendación $\mathrm{N}^{\circ} 163$ por su parte contiene disposiciones que sirvan de modelo para el contenido de acuerdos bilaterales o multilaterales de seguridad social. Finalmente en relación a la conservación de derecho en materia de seguridad social el Convenio $\mathrm{N}^{\circ} 128$, contiene una cláusula específica sobre esta materia.

A continuación incluimos un cuadro en que se indica el número de ratificaciones de los principales instrumentos de la OIT relativos a los migrantes y a la protección de seguridad social de estos. Además, se incluye si estos instrumentos han sido ratificados por Chile o no.

\begin{tabular}{l|l|l}
\hline \multicolumn{1}{c|}{ Convenio } & \multicolumn{1}{|c}{ Ratificaciones } & \multicolumn{1}{c}{ Chile } \\
\hline Convenio $\mathrm{N}^{\circ} 19$ & 121 países & 8 Oct. 1931 \\
\hline Convenio $\mathrm{N}^{\circ} 48$ & 12 países (4 denuncia) & No ratificado \\
\hline
\end{tabular}




\begin{tabular}{l|l|l}
\hline \multicolumn{1}{c|}{ Convenio } & \multicolumn{1}{|c}{ Ratificaciones } & \multicolumn{1}{c}{ Chile } \\
\hline Convenio $N^{\circ} 102$ & 48 países & No ratificado \\
\hline Convenio $N^{\circ} 118$ & 38 países (1 denuncia) & No ratificado \\
\hline Convenio $N^{\circ} 157$ & 4 países & No ratificado \\
\hline
\end{tabular}

Fuente : Ilolex visitado el 28 de noviembre de 2012.

\subsection{PRINCIPIOS LEGALES DE PROTECCIÓN DEL MIGRANTE}

Existe una serie de principios internacionalmente aceptados que constituyen el marco general para la celebración de acuerdos bilaterales o multilaterales que regulan la coordinación de sistemas de seguridad social ${ }^{40}$. Como señala Gumucio:

"En lo que atañe a la seguridad social, la coordinación de legislaciones para atender los problemas de los migrantes supone la aceptación de los principios e instrumentos tradicionales que generalmente orientan los convenios bilaterales y multilaterales de seguridad social y que han sido recogidos en los convenios de la OIT"41.

Los principios básicos de las normas de coordinación de los sistemas de seguridad social son considerados como la columna vertebral para los acuerdos bilaterales y multilaterales que regulan esta materia. Estos principios que se han deducido de las normas de la OIT varían en su número dependiendo del autor que se trate. A continuación analizaremos brevemente cada uno de los principios que consideramos forman parte de esta columna vertebral $^{42}$ :

a) Igualdad de trato, ello significa que el trabajador migrante debe tener, tanto como fuese posible, los mismos derechos y obligaciones que los nacionales del país de destino. La igualdad de trato implica entregar beneficios con independencia de la nacionalidad del beneficiario. Este principio también ha sido interpretado como una prohibición de toda discriminación por razón de la nacionalidad. De esta manera, bajo este principio podemos encontrar la idea del principio de igualdad de trato y no discriminación por nacionalidad del beneficiario.

b) Determinación de la legislación aplicable para asegurar, mediante el establecimiento de reglas de determinación de legislación aplicable, que la seguridad social del trabajador migrante se encuentra regida todo el tiempo por la legislación de un solo país. La idea detrás de este principio es evitar cualquier conflicto de posibles leyes

\footnotetext{
40 Sobre los principios ver: Hirose, Kenichi; NikaC, Milos y TAmagno, Edward, op. cit. (n. 5). Cifuentes Lillo, Hugo. "Tratados internacionales de seguridad social: Convenios bilaterales", en: Estudios en Homenaje al profesor William Thayer. Santiago, Chile: Sociedad Chilena de Derecho del Trabajo y Seguridad Social, 1998, p. 22 y ss. OIT. Introducción a la seguridad social. Oficina Internacional del Trabajo, Ginebra, 1984.

41 Gumucio R, J., op. cit. (n. 29), p. 59.

42 Nos basaremos principalmente en lo enunciado en Hirose, Kenichi; NiKaC, Milos y TAmagno, Edward, op. cit. (n. 5), p. 8. Ello sin perjuicio de completar lo allí descrito con explicaciones propias.
} 
aplicables así como también evitar el vacío de la legislación aplicable. Así como en el Derecho Internacional Privado, solo una legislación de un país se aplicará a la vez. En cuanto a la determinación de la legislación aplicable se utiliza el principio básico de lex loci laboris, se aplica la legislación en donde la prestación de servicios del contrato de trabajo haya sido prestada.

Resulta interesante destacar en este principio, las situaciones que se presenten en caso de la cada vez más frecuente en el mundo puesta a disposición de trabajadores y la de los trabajadores fronterizos. En el primer caso, se trata de un excepción a la regla que la legislación de seguridad social es la del país anfitrión en donde se ejercen la prestación de servicios. En este caso es la legislación del país de origen que sigue al trabajador migrante durante la vigencia de la puesta a disposición. En el segundo caso, la regla también puede verse alterada y la solución generalmente ha sido encontrada a través de tratados bilaterales, ya sea que reglen la coordinación entre las dos legislaciones o establezcan reglas particulares para las localidades fronterizas de dos países.

c) Mantenimiento de los derecho adquiridos, esto significa que todo derecho adquirido, o en curso de adquisición, debe ser garantizado para el migrante en un territorio. Incluso si ha sido adquirido en el territorio de otro Estado. No deben existir restricciones para su pago, en ninguno de los países involucrados, de las prestaciones para las cuales el trabajador migrante haya calificado. De esta manera no se pierden las prestaciones por el hecho de trasladarse a otro país. Algunos autores han descrito este principio con el término exportabilidad o portabilidad de beneficios sociales para hacer referencia a la misma idea. El mantenimiento de los derechos a las prestaciones resultará distinto de acuerdo con el mecanismo de financiamiento y al riesgo de que se trate. Los pagos periódicos de prestaciones a largo plazo como pensiones de vejez tienen un mecanismo diferente a aquellas prestaciones de pagos periódicos de corto plazo, como el desempleo. Esto es debido a que responden a lógicas diferentes. También el hecho que la prestación sea contributiva o no contributiva tiene incidencia en la forma en como se establecerá el mantenimiento de derechos.

d) Mantenimiento de derechos en curso de adquisición, esto significa que donde un derecho esté condicionado al cumplimiento de un período de calificación se debe tomar en cuenta los periodos completados por el migrante en cada país. De esta manera se agregarán o sumarán todos los períodos de cotización, de residencia o de afiliación para la obtención de la prestación. Esta técnica además se la ha denominado como de totalización de los periodos. Para esto se puede notar diferentes técnicas: cálculo proporcional, cálculo directo o integración.

e) Colaboración administrativa y técnica entre instituciones nacionales con el objeto de facilitar la gestión a los beneficiarios. La comunicación fluida y completa de información entre las instituciones a cargo del otorgamiento de beneficios resulta clave para que la coordinación cumpla con sus objetivos. 
f) Reciprocidad, es un principio que se encuentra subyacente en las convenciones de la OIT y significa que cada país parte del acuerdo debe aplicar los mismos mecanismos para hacer su propia seguridad social más accesible a los trabajadores migrantes. La reciprocidad implica un razonable grado de comparación en las obligaciones que tenga cada país en el acuerdo. Este principio es la base de la negociación de coordinación. Así, implica una voluntad política para que la técnica jurídica se acerque entre ambos países. Voluntad que resulta vital para otorgarle protección de seguridad social al trabajador migrante.

\subsection{MECANISMOS DE COORDINACIÓN: CONVENIOS BILATERALES Y MULTILATERALES DE SEGURIDAD SOCIAL}

Los mecanismos que pueden entregar continuidad a las prestaciones de seguridad social del trabajador migrantes pueden ser unilaterales, bilaterales y multilaterales. Tan solo las bilaterales y multilaterales implican un cierto grado de coordinación. Aquellas medidas unilaterales son aquellas que adoptan el estado anfitrión o el estado enviante de migrantes a fin de proteger a los migrantes en su territorio o a sus nacionales en el extranjero.

Los convenios de seguridad social bilaterales y multilaterales se celebran bajo la premisa los trabajadores solo tendrán que pagar cotizaciones en dos países para cubrir el mismo riesgo y que a su vez no pierdan los periodos cotizados cuando se desplazan a otro país. De esta manera, estos acuerdos de seguridad social van a contener disposiciones para una o varias ramas de la seguridad social de los países involucrados o se referirán al sistema en su conjunto. Para poder lograr la cobertura pretendida, se ha entendido que deben cumplir con 5 objetivos ${ }^{43}$ : igualdad de trato, pago de beneficios en el extranjero, determinación de la legislación aplicable mantenimiento de derechos en curso de adquisición y asistencia administrativa. No confundir con los principios de la coordinación aquí hacemos referencia a los objetivos que deben cumplir los acuerdos sobre seguridad social en orden a cumplir con el objeto de entregar continuidad de la cobertura.

Como se puede conceptualizar a este tipo de acuerdos de seguridad social. Según el profesor Cifuentes:

"Entendemos a los convenios de seguridad social, como pactos o instrumentos de carácter internacional, suscritos por dos países, salvo que se trate de un convenio multilateral, que buscan resolver todos o parte de los requerimientos de seguridad social que pueden afectar a los trabajadores migrantes. Por esos tratados se persigue la mantención de la eficacia y oportuno reconocimiento de los periodos de afiliación que se han configurado en diferentes países, ya sea, cotizando, trabajando o simplemente residiendo, para dar lugar, en la oportunidad legal correspondiente, al derecho a una pensión por vejez, invalidez, sobrevivencia, muerte, prestaciones por salud, desempleo u otros, en un sistema de seguridad social especifico" ${ }^{44}$.

43 Hirose, Kenichi; Nikac, Milos y Tamagno, Edward, op. cit. (n. 5), p. 25.

44 Cifuentes Lillo, Hugo. “Tratados internacionales... (n. 40), p. 21. 
Por su parte, un grupo de expertos internacionales en seguridad social han señalado que:

"un acuerdo de seguridad social coordina los esquemas de seguridad social de dos o más países para así poder superar, sobre la base de la reciprocidad, las barreras que puedan de otra manera evitar que los trabajadores migrantes y a los miembros de sus familias de recibir prestaciones de los sistemas de cualquiera de los países en los cuales ha trabajado" 45 .

Como podemos constatar el objeto principal de estos acuerdos es establecer un mecanismo de coordinación, bilateral o multilateral. Pero además,

"generalmente, los acuerdos multilaterales son considerados por tener la ventaja de generar estándares y reglamentaciones comunes, evitando así discriminación entre migrantes de varios países enviantes que de otra manera pueden obtener diferentes derechos y prestaciones por la vía de acuerdos bilaterales. Por otro lado, los acuerdo bilaterales tienen la ventaja de proveer gran flexibilidad y la posibilidad de tomar en cuenta situaciones específicas de los países involucrados. Además, se requiere menos tiempo y procedimiento para alcanzar a un acuerdo entre las dos partes. Por estas razones, los acuerdos bilaterales son ampliamente más usados para la coordinación de sistemas de seguridad social" 46 .

A través del mundo se han celebrado una cantidad importante de acuerdos en materia de seguridad social aunque su número podemos considerarlo insuficiente ${ }^{47}$. La tendencia hoy en día es la implementación de mecanismos de coordinación regionales que permitan una mayor integración. Estos intentos regionales se presentan en diversas partes del globo ${ }^{48}$.

Los pioneros en esta materia fueron las Comunidades Europeas evolucionando desde primitivas reglamentaciones hasta los recientes reglamentos 883/2004 y 987/2009 ${ }^{49}$ de la Unión Europea. La reglamentación europea constituye un entramado complejo que permite entregar continuidad a las prestaciones de seguridad social de modelos de estado de bienestar diferentes dentro de Europa. Coordina prestaciones contributivas como no contributivas

\footnotetext{
45 Hirose, Kenichi; Nikac, Milos y TAmagno, Edward, op. cit. (n. 5), p. 19.

46 Hirose, Kenichi; Nikac, Milos y Tamagno, Edward, op. cit. (n. 5), p. 4.

47 Para informarse sobre los acuerdos celebrados a través del mundo por cada país ver la base de datos Natlex de la OIT en http://www.ilo.org/dyn/natlex/natlex browse.home

48 Sobre los ejemplos de acuerdo multilaterales regionales ver: Hirose, Kenichi; NiKaC, Milos y TAMAgno, Edward, op. cit. (n. 5), p. 49.

49 Sobre la coordinación europea ver SÁNCHEZ-RodAS, C. (dir.). La coordinación de los sistemas de Seguridad Social. Los reglamentos 883/2004 y 987/2009. Murcia, España: Laborum, 2010. JORENS, Yves (Ed). 50 years of social security coordination. Past-Present-Future, Report of the conference celebrating the 50th Anniversary of the European Coordination of Social Security. European Commission, Directorate General for Employment, Social Affairs and Equal Opportunities, Publication Office of the European Union, 2010. MAUDET-BENDAHAN, Marilyn, op. cit. (n. 1).
} 
de todas las ramas de la seguridad social. Es sin lugar a dudas el modelo a seguir tanto por su detalle como por su ámbito de aplicación ${ }^{50}$.

La región latinoamericana por su parte ha tenido una serie de intentos de coordinación región y subregional ${ }^{51}$. Algunos con más éxitos que otros. Podemos destacar la existencia de mecanismos de coordinación presentes en el Mercosur ${ }^{52}$, Caricom ${ }^{53}$ y también en la Comunidad Andina ${ }^{54}$. Sin embargo, el reciente Convenio Multilateral Iberoamericano de Seguridad Social ${ }^{55}$ ha llevado la implementación de mecanismos de coordinación en la región a un nivel superior. Ha significado un importante avance hacia una coordinación regional sin que la región tenga una unión política (se incluye además a Portugal y España).

En África, región con la cual Latinoamérica comparte muchas características de su mercado de trabajo, no se ha logrado implementar acuerdos regionales de coordinación con éxito. La Conferencia Interafricana de Previsión Social (Conférence Interafricaine de la Prévoyance Sociale), CIPRES, constituye la reunión de 15 países de habla francesa. En 1996, adoptaron un Convenio Multilateral de Seguridad Social del CIPRES (Convention Multilatérale de Sécurité Sociale) siendo un acuerdo sin precedentes para la región y el mundo sobre la materia. No obstante que un grupo de países lo ha ratificado, este convenio aún no entre en vigor por falta del número suficiente de ratificaciones para ello. Por otro lado, en el mismo continente, la Comunidad para el Desarrollo Sudafricano (SADC) ${ }^{56}$ se encuentra desde hace ya algún tiempo estudiando la posibilidad de adoptar algún tipo de instrumento que permita alcanzar los objetivos de la coordinación ${ }^{57}$.

En Asia y en Oceanía no existen por el momento acuerdos regionales en materia de coordinación de sistemas de seguridad social. Sin embargo, en esta región países como Fili-

50 Para una comparación entre los mecanismos europeos y latinoaméricanos ver ARELLANO OrTiZ, Pablo; BRIGANTI, Francesco. "Europa y América Latina: encuentros y desencuentros en la búsqueda de la coordinación de los sistemas de seguridad social”, en: Programa de Estudios Europeos Universidad de Concepción. Europa y América Latina. Dos caminos, ¿un destino común? Santiago, Chile: RIL Editores, 2012, pp. 125-155. Para un informe sobre los modelos sociales ver: MINISTERIO de Trabajo y Asuntos Sociales, Subsecretaría de Estado de la Seguridad Social, España. "El modelo social europeo y los proceso de reforma de la seguridad social en los países latinoamericanos". Conferencia de Ministros de Seguridad Social de Europa y Latinoamérica, Valencia, 13, 14 y 15 de mayo de 2002.

51 Al respecto: ARELlano OrTIZ, Pablo. "New developments in social security protection for migrants in Latin-American region". Presentado en la Conferencia "Acces Denied. Working on a new paradigm, International conference on social protection and migration" organizada por la Universidad de Amsterdam, Holanda, realizada los días 13 y 14 de marzo de 2012.

52 Más detalles sobre este acuerdo subregional en http://www.mercosur.int/ . Sobre su eventual relación con Chile ver: GUMUCIO R, J., op. cit. (n. 29), pp. 58-64.

53 Más detalles sobre este acuerdo regional en http://www.caricom.org/index.jsp

54 Más detalles sobre este acuerdo subregional en http://www.comunidadandina.org/

55 Al respecto ver www.oiss.org. Además, ver al respecto: ARELlANO ORTIZ, Pablo; BRIGANTI, Francesco. "Europa y América Latina..., (n. 50). Arellano OrTiZ, Pablo. "New developments... (n. 51). SÁnChEZ-RodAs, C. "El Convenio Multilateral Iberoamericano de seguridad Social”, en: Revista General de Derecho del Trabajo y de la Seguridad no 26, junio, Madrid, España, 2011. JimÉNEZ, Adolfo. "Una experiencia inédita", en: Miradas al Exterior, Revista de Información Diplomática del Ministerio de Asuntos Exteriores y de Cooperación de España, no 18, abril-junio, p. 19.

56 Sobre esta comunidad ver http://www.sadc.int/

57 OLIVIER, Marius. "Social security developments in the SADC region and future prospects for coordination". Trabajo presentado en el seminario "Seguridad social y trabajadores migrantes", realizado el día 24 de septiembre 2012 - Auditorio 4, Edificio Nahmías, U. Austral de Chile, Campus Isla Teja, organizado por la Facultad de Ciencias Jurídicas y Sociales de la Universidad Austral de Chile. 
pinas han optado por adoptar medidas unilaterales ${ }^{58}$. Así, si un nacional filipino emigra la legislación de su país lo protege en el extranjero. Este tipo de medidas si bien no son puramente de coordinación también implican la obtención de la continuidad de las prestaciones de seguridad social. Constituyen además una tendencia importante en las recientes medidas adoptadas por diversos países alrededor del mundo.

\subsection{REFLEXIONES SOBRE LOS ASPECTOS INTERNACIONALES}

Resulta difícil concluir de manera tajante hacia dónde va la tendencia en materia de instrumentos internacional que permita la continuidad de las prestaciones de seguridad social. Las realidades son muy diferentes en las distintas regiones del planeta. Claramente, no hay ninguna panacea que resuelva el desafío de cubrir al trabajador migrante ${ }^{59}$.

Compartimos la evaluación del profesor Cifuentes:

"los convenios de seguridad social de carácter bilateral, en la mayoría de los casos resuelven la situación previsional de los migrantes en materia de pensiones de vejez, invalidez y sobrevivencia en regímenes de carácter contributivo. Por consiguiente, queda un amplio espectro de prestaciones que no son consideradas o, si lo son, es de manera fragmentaria y para determinados grupos, lo que afecta la consecución del principio de universalidad en sus dos vertientes, subjetiva y objetiva" ${ }^{60}$.

Los convenios bilaterales no entregan una protección universal objetiva. En un número importante de casos están destinados solo a reglamentar materias relativas a las pensiones de vejez. Estimamos, que los acuerdos bilaterales podrían cubrir todos los riesgos sociales realizando así una verdadera coordinación entre dos legislaciones.

Consideramos que falta una aproximación más completa a la cobertura del trabajador migrante. Hemos sostenido que esta debe ser coherente ${ }^{61}$, intentando cubrir se todas las categorías de trabajadores migrantes y a su vez que se les cubran todos los riesgos sociales. Son los tratados multilaterales los que siguiendo el ejemplo europeo buscan esta aproximación más coherente y completa. Así, se puede señalar que los tratados multilaterales tienen la ventaja de facilitar la carga administrativa y, que por su lado, los acuerdos bilaterales pueden ser el primer paso hacia un acuerdo multilateral más complejo ${ }^{62}$. Sin embargo como veremos luego, el único convenio multilateral en vigor que sigue la tendencia europea, Convenio Multilateral Iberoamericano de Seguridad Social, no cubre todas las ramas de la seguridad social. Este posee un ámbito de aplicación materia limitado. Cubriéndose los pagos periódicos de regímenes contributivos: invalidez, vejez, sobrevivientes, accidentes y enfer-

\footnotetext{
58 Hirose, Kenichi; NiKaC, Milos y TAMAgno, Edward, op. cit. (n. 5), p. 14.

59 Hirose, Kenichi; Nikac, Milos y TAMagno, Edward, op. cit. (n. 5), p. 15.

60 Cifuentes Lillo, Hugo. "Tratados internacionales... (n. 40), p. 33.

61 Arellano Ortiz, Pablo. "Protección de seguridad social para los migrantes: la necesidad de una intervención legal coherente". Trabajo presentado en el seminario "Seguridad social y trabajadores migrantes", realizado el día 24 de septiembre 2012 - Auditorio 4, Edificio Nahmías, U. Austral de Chile, Campus Isla Teja, organizado por la Facultad de Ciencias Jurídicas y Sociales de la Universidad Austral de Chile.

62 Hirose, Kenichi; NiKaC, Milos y Tamagno, Edward, op. cit. (n. 5), p. 39.
} 
medades profesionales. Si bien se excluyen todo tipo de prestaciones en especie, y, por ende las prestaciones médicas, se estipula que los países signatarios pueden incluirlas a través de un acuerdo para tal efecto. Así, se puede colegir que los instrumentos internacionales poseen una ambición a veces muy amplia pero limitada a algunos riesgos para algunos trabajadores. Resulta necesario poder encauzar los esfuerzos para extender la protección hacia nuevas categorías de trabajadores y a todos los riesgos sociales.

Finalmente, podemos señalar que actualmente los instrumentos internacionales han evolucionado enormemente en la protección de seguridad social del trabajador migrante. Sin embargo, frente a la globalización y a la complejización de las relaciones de trabajo, se deben aunar aún más los esfuerzos en vista de una efectiva continuidad y coherente protección de los riesgos sociales del trabajador migrante.

\section{BIBLIOGRAFÍA}

Arellano Ortiz, Pablo. Universalimo e Individualismo en el derecho chileno de pensiones. Santiago, Chile: Editorial Librotecnia, 2012.

ARELLANO ORTIZ, Pablo. "La cobertura de los accidentes del trabajo y enfermedades profesionales por las normas internacionales del trabajo de la OIT", en: Revista Chilena de Derecho de Trabajo y de la Seguridad Social, vol. 2, n 3, Santiago, Chile, 2012, pp. 163-180.

ARELlano OrTIZ, Pablo. "New developments in social security protection for migrants in Latin-American region". Presentado en la Conferencia "Acces Denied. Working on a new paradigm, International conference on social protection and migration" organizada por la Universidad de Amsterdam, Holanda, realizada los días 13 y 14 de marzo de 2012.

ARELlano OrTIZ, Pablo. "Protección de seguridad social para los migrantes: la necesidad de una intervención legal coherente". Trabajo presentado en el seminario "Seguridad social y trabajadores migrantes", realizado el día 24 de septiembre 2012 - Auditorio 4, Edificio Nahmías, U. Austral de Chile, Campus Isla Teja, organizado por la Facultad de Ciencias Jurídicas y Sociales de la Universidad Austral de Chile.

Arellano OrTiz, Pablo. "La conformidad de la legislación chilena a las normas internacionales del trabajo de la OIT", en: Revista de Derecho de la Universidad Católica de la Santísima Concepción, Concepción, Chile, 2011, pp. 39-60.

Arellano Ortiz, Pablo; Briganti, Francesco. "Europa y América Latina: encuentros y desencuentros en la búsqueda de la coordinación de los sistemas de seguridad social”, en: PROGRAMA DE EsTUDIOS EUropeos Universidad De CONCEPCIÓn. Europa y América Latina. Dos caminos, ¿un destino común? Santiago, Chile: RIL Editores, 2012, pp. 125-155.

Cifuentes Lillo, Hugo. “Tratados internacionales de seguridad social: Convenios bilaterales”, en: Estudios en Homenaje al profesor William Thayer. Santiago, Chile: Sociedad Chilena de Derecho del Trabajo y Seguridad Social, 1998, pp. 16-38.

Cifuentes Lillo, Hugo. "Seguridad Social: Conceptos, definiciones y sistema de protección social en Chile", en: Homenaje el profesor Alfredo Bowen H. Anuario de derecho del Trabajo y Seguridad Social n ${ }^{\circ} 1$. Santiago, Chile: Sociedad Chilena de Derecho del Trabajo y de la Seguridad Social, 2000, pp. 9-22.

Cifuentes Lillo, Hugo; ARellano OrTiz, Pablo. "Derecho a la Seguridad Social y la protección por pensiones de vejez, invalidez y sobrevivencia en Chile”, en: ROJAS MIÑO, Irene (Coordinadora). Cincuenta años de la Sociedad Chilena de Derecho del Trabajo y de la Seguridad Social: Homenaje. Santiago, Chile: Sociedad Chilena de Derecho del Trabajo y de la Seguridad Social, Abeledo Perrot LegalPublishing, 2011, pp. 177-288. 
Comisión Mundial sobre la Dimensión Social de la Globalización. Por una globalización justa: crear oportunidades para todos. Ginebra, Suiza, OIT, 2004.

Coursier, Philippe. Conflit de lois en matière de contrat de travail. Bibliothèque de droit privé, tome 230. París, Francia: L.G.D.J, 1993.

Gumucio R, J. "Chile en el Mercosur: armonización de sistemas de Seguridad Social”, en: Revista Laboral Chilena, enero, Santiago, Chile, 1997, pp. 58-64.

Hirose, Kenichi; Nikac, Milos y TAMAGNO, Edward. Social security for migrant workers. A right-based approach. International Labour Organization, Decent Work Technical Support Team, and Country Office for Central and Eastern Europe-Budapest, ILO, 2011.

Humblet, M.; SILVA, R. Normas para el siglo XXI, Seguridad Social. Ginebra, Suiza, OIT, 2002.

Humeres Noguer, Héctor. Derecho del Trabajo y de la Seguridad Social. Tomo III. Derecho de la Seguridad Social. $-17^{\text {a }}$ edición ampliada y actualizada-. Santiago, Chile: Editorial Jurídica de Chile, 2005.

JAVILLIER, Jean-Claude; GERNIGON, Bernard (sous la direction de). Les normes internationales du travail: un patrimoine pour l'avenir. Mélanges en l'honneur de Nicolas Valticos. Geneva, Italie: Bureau International du Travail, 2004.

JimÉNEZ, Adolfo. "Una experiencia inédita”, en: Miradas al Exterior, Revista de Información Diplomática del Ministerio de Asuntos Exteriores y de Cooperación de España, no 18, abril-junio.

JORENS, Yves (Ed). 50 years of social security coordination. Past-Present-Future, Report of the conference celebrating the 50th Anniversary of the European Coordination of Social Security. European Commission, Directorate General for Employment, Social Affairs and Equal Opportunities, Publication Office of the European Union, 2010.

KulKe, U.; CiCHON, C.; PALK, K. "Changing tides: a revival of a right-based approach to social security”. En: LANGEndonCK, J. van (Ed.). The Right to Social Security. Inglaterra, Londres: Intersentia, AntwerpOxford, 2007.

KUPTSCH, C. «La privatisation dans la sécurité sociale: un slogan aux multiples facettes- Remarques sur le concept de privatisation en fonction du contexte», In: SCHEIL-ADLUNG, X. Construire la sécurité sociale: la privatisation en question. Francia: AISS, Peter Lang SA, Bern, 2001.

LARoque, P. «De l'assurance à la Sécurité Sociale, l'expérience française», en: Rev. Inter. Trav. vol. LVII, nº 6, France, París, 1948, pp. 623 et. ss.

LYON-CAEN, Gerard y Antoine. Droit social européen et international. -8 édition-. Paris, France: Précis, Dalloz, 1993.

LyOn-CAEN, A.; URBAN, Q. (dir). Le droit du travail á l'épreuve de la globalisation. Dalloz "Thémes et commentaires", Paris, 2008.

Martínez Pizarro, J. (Editor). Migración internacional en América Latina y el Caribe. Nuevas tendencias, nuevos enfoques. Santiago, Chile: Cepal, United Nations, 2011.

MAUDET-BENDAHAN, Marilyn. La mobilité géographique du travailleur salarié au sein de l'Union européenne. Bibliothèque de droit social, Tome 57. Paris, L.G.D.J, 2012.

Ministerio de Trabajo y Asuntos Sociales, Subsecretaría de Estado de la Seguridad Social, EspaNA. "El modelo social europeo y los procesos de reforma de la seguridad social en los países latinoamericanos". Conferencia de Ministros de Seguridad Social de Europa y Latinoamérica, Valencia, 13, 14 y 15 de mayo de 2002.

Moreau, Marie-Ange. Normes sociales, droit du travail et mondialisation-Confrontations et mutations, A droit ouvert. Dalloz-Sirey, Paris, 2006.

Novoa Fuenzalida, Patricio. Derecho de la Seguridad Social. Santiago, Chile: Editorial Jurídica de Chile, 1977. 
Pablo Arellano Ortiz / Trabajadores migrantes y seguridad social: aproximación nacional e internacional a los mecanismos de protección...

OLIVIER, Marius. "Social security developments in the SADC region and future prospects for coordination". Trabajo presentado en el seminario "Seguridad social y trabajadores migrantes", realizado el día 24 de septiembre 2012. Auditorio 4, Edificio Nahmías, U. Austral de Chile, Campus Isla Teja, organizado por la Facultad de Ciencias Jurídicas y Sociales de la Universidad Austral de Chile.

OIT. Seguridad social para la justicia social y una globalización equitativa. Discusión recurrente sobre la protección social (seguridad social) en virtud de la Declaración de la OIT relativa a la justicia social para una globalización equitativa, Informe VI, Sexto punto del orden del día, Conferencia Internacional del Trabajo, 100.a reunión, 2011 .

OIT. En busca de un compromiso equitativo para los trabajadores migrantes en la economía globalizada. Informe VI, Sexto punto del orden del día Conferencia Internacional del Trabajo, 92a reunión, 2004.

OIT. Seguridad social: un nuevo consenso. Ginebra, 2002.

OIT. Las normas internacionales del trabajo, Un enfoque global. Ginebra, 2002.

OIT. Rapport sur le travail dans le monde 2000: sécurité du revenu et protection sociale dans un monde en mutation. Commission de l'emploi et de la politique sociale. Conférence Internationale du Travail, $79^{\circ}$ session, Genève, 2000.

OIT. Trabajo Decente. Memoria del Director General, Conferencia Internacional del Trabajo, 87a reunión, Ginebra, 1999.

OIT. Introducción a la seguridad social. Oficina Internacional del Trabajo, Ginebra, 1984.

Rodríguez-PiÑero Bravo Ferrer, Miguel. “La OIT y los trabajadores migrantes”. En: JaVillier, Jean-Claude; GERNIGON, Bernard (sous la direction de). Les normes internationales du travail: un patrimoine pour l'avenir. Mélanges en l'honneur de Nicolas Valticos. Geneva, Italie: Bureau International du Travail, 2004, pp. 541-564.

SÁnCHEZ-RODAS, C. "El Convenio Multilateral Iberoamericano de seguridad Social”, en: Revista General de Derecho del Trabajo y de la Seguridad no 26, junio, Madrid, España, 2011.

SÁNCHEZ-RODAS, C. (dir.). La coordinación de los sistemas de Seguridad Social. Los reglamentos 883/2004 y 987/2009. Murcia, España: Laborum, 2010.

SERVAIS, Jean Michel. Normes Internationales du Travail. Paris, France: L.G.D.J, 2004.

TARAN, Patrick; Geronimi, Eduardo. “Globalización y migraciones laborales: importancia de la protección”, en: Perspectivas sobre las migraciones laborales. 3 S, Programa de Migraciones Internacionales, Oficina Internacional del Trabajo, Ginebra, 2003.

VAlTiCos, Nicolas. Droit International du Travail. -2e édition-. Dalloz, 1983.

VIJ, R. (ed). Globalisation and Werlfare, a critical reader. Palgrave Macmillan, 2007.

VitTin-BALMA, C. “Trabajadores Migrantes”, en: OIT. Las normas internacionales del trabajo. Un enfoque global. Ginebra, Suiza, 2002, pp. 130- 171. 